\title{
Enabling Conditional Cross-Domain Data Sharing via a Cryptographic Approach
}

\author{
Giuseppe Bianchi \\ CNIT / Univ. Roma Tor Vergata \\ Via del Politecnico 1, 00133 Roma \\ Email: giuseppe.bianchi@uniroma2.it
}

\author{
Hanieh Rajabi \\ CNIT / Univ. Roma Tor Vergata \\ Via del Politecnico 1, 00133 Roma \\ Email: hanieh.rajabi@uniroma2.it
}

\author{
Marco Sgorlon \\ CNIT / Univ. Roma Tor Vergata \\ Via del Politecnico 1, 00133 Roma \\ Email: marco.sgorlon@gmail.com
}

\begin{abstract}
Cross-domain Internet-scale collaborative security is affected by a native dichotomy. On one side, sharing of monitoring data across domains may significantly help in detecting large scale threats and attacks; on the other side, data sharing conflicts with the need to protect network customers' privacy and confidentiality of business and operational information. The approach first proposed in this paper enables what we call "conditional data sharing", i.e., permit cross-domain sharing of fine-grained organized subsets of network security data (called monitoring data feeds), only when a threshold number of domains are ready to reveal their data for the same feed. The proposed approach revolves on a careful combination of distributed threshold based cryptography with identity-based encryption. It appears scalable and easy to deploy, not requiring neither a-priori monitoring data feeds identification, nor explicit coordination among domains. Protection is accomplished by "simply" using different cryptographic keys per feed, and automatically permitting perfeed key reconstruction upon the occurrence of independent and asynchronous per-domain/per-feed alerts.
\end{abstract}

\section{INTRODUCTION}

Internet-scale security data sharing is a controversial and challenging problem, founded on a natural dichotomy.

On one side, it is widely perceived [1], [2], [3] that sharing of monitoring information across domains may significantly improve the ability to detect and mitigate global threats and respond to crises. The case for cooperative interdomain network monitoring is getting even stronger with the ever increasing deployment of large-scale coordinated attach infrastructures such as Botnets [4]. Against such cross-domain threats, detecting an emerging attack from a single domain's observation point may be very challenging, whereas crossdomain harvesting and exchange of network security data, enabling correlation of monitoring information from multiple points, may make the diagnosis of an attack or anomaly much faster and simpler.

On the other side, at least two major concerns prevent the sharing of monitoring data across domains. First, monitoring data contain plenty of personal data concerning the network domain's customers. Indeed, customers' privacy can hardly coexist with any form of massive packet or flow trace collection, since even header information such as IP addresses are to all extents classified as personal data by existing data protection legislation in Europe [5], [6]. And the extensive documentation on the subtleties, complexities, and risks behind IP address anonymization [7], with the evidence of many de-anonymization attacks [8], [9], raise severe concerns about the effectiveness of anonymization approaches in terms of privacy preservation [10]. Second, sharing of monitoring information across (competitor) domains should not disclose business critical information. This is a particularly critical requirement, as operational information to be protected may include traffic type, volumes, and meta-information which may reveal the domain's performance, number and location of vantage points, network topology information, and so on; even the mere fact that the domain is under attack may be in some cases considered an operational secret.

\section{Conditional Data Sharing}

We believe that sharing agreements among operators, albeit necessary, are not sufficient to convincingly address the above highlighted issues; further technical advances are required in the ability to protect and carefully control the actual sharing of data. This paper contributes in this direction by introducing and technically supporting an approach which we call conditional data sharing.

The idea is to selectively share fine-grained organized network security data related to some critical event, traffic behaviour, entity, or phenomenon, if and only if other collaborative partners have observed the same criticality. In other words, cross-domain monitoring data sharing gets conditioned to the detection of relevant anomalies or events in multiple domains. The underlying rationale is that a legitimate Internet scale collaborative analysis should aim at discovering global trends and threats affecting multiple domains, whereas monitoring information of just local interest should not be shared.

To the best of our knowledge, the above problem statement has been originally posed in [1], who challenged the research community to "design efficient distributed protocols and similarity metrics for network security data to ensure that each contributor only reveals the data if a threshold number of other participants are ready to reveal similar data".

In this paper we provide a preliminary answer to this challenge, through a carefully constructed cryptographic approach. Our basic idea to is export all possible data of interest, but protect it through encryption, thus making it unaccessible by other domains. Different automatically generated encryption keys are used for a potentially huge multiplicity of fine-grained organized monitoring data streams which, in most general- 
ity, we call feeds. The core of our proposed cryptographic construction consists in permitting participating domains to decrypt a specific feed only when they recover the relevant key. This is accomplished by means of a fully distributed process which permits per-feed key reconstruction only when a minimum number of domains provide relevant cryptographic material.

\section{Why a Purely Cryptographic Approach?}

We do not claim that a cryptographic approach is strictly needed. Indeed, the conditional data sharing functionality introduced in this paper may probably be alternatively implemented via a specifically devised multi-party collaborative data querying protocol: any domain suspecting that some given feed is anomalous may contact the other domains and verify whether this is the case from their perspective as well.

However, even besides the non trivial technical hurdles emerging in setting up such a protocol, with its relevant finegrained authorization primitives involving conditions such as agreement among a threshold number of parties, in our opinion three reasons favour a cryptographic approach.

A first one is that handling of fine-grained per-feed queries may become unmanageable as the number of feeds and frequency of inter-domain contacts increases, and when feeds are a-priori unknown and may start/appear at any time. In contrast, our cryptographic approach permits autonomous and asynchronous operation among domain (each domain releases the encrypted data independently from others), and the only signalling required is done once for all at setup.

A second reason is that the proposed fully automated, non interactive, approach may be deployed also over trans-national monitoring infrastructures ${ }^{1}$ composed of "just" passive monitoring sensors, with very high performance requirements, lack of long-term storage capabilities, and not meant to directly interact each other. Indeed, some early ideas in this direction have been anticipated in [11] and demonstrated feasible at gbps speed in [12] (although for just the single-probe scenario and for a quite different and simpler technical solution).

Finally, a third reason is that the security of the proposed cooperation scheme does not depend on any server infrastructure, but relies on the security of the employed cryptographic approaches. Obviously this is a quite debatable argument, to be interpreted as a personal opinion of the authors - albeit supported by several cases in the history of network security protocols (e.g. SSL/TLS) which suggest that the majority of the past security vulnerabilities emerged because of shortcomings in the protocol specification (or implementation) rather than weaknesses in the employed cipher suites.

\section{Scenario, Definitions, Problem Statement}

Our reference scenario consists of $n$ independent administrative domains $D_{1}, \ldots, D_{n}$. For simplicity, we assume

\footnotetext{
${ }^{1}$ an example being the advanced pilot European Internet traffic monitoring infrastructure deployed by a past European project LOBSTER - Large Scale Monitoring of Broadband Internet Infrastructures (LOBSTER), www.istlobster.org, as well as follow ups and similar initiatives outside EU.
}

that the number of cooperating parties is fixed and known beforehand. No central coordination entity or third party authority is assumed: indeed approaches mandating a coordinating third party would hardly fit with the current multidomain networking business context.

Each domain is, as usual, in charge to monitor just its internal network. Cross-domain cooperation restricts to the possibility of sharing some monitoring data organized as discussed below; more advanced forms of cooperation, such as cross-domain threat mitigation, are deemed to be less viable and are out of the scopes of this paper.

\section{Monitoring Data Feeds}

Our proposed approach does not depend on which specific data is gathered by the internal monitoring tasks, nor it poses any restriction on the type and amount of data candidate to be shared. The only requirement is that each domain is able to organize the shareable monitoring data into an arbitrary multiplicity of named logs or streams.

More precisely, we introduce, and adopt throughout the paper, the following notation. We define a monitoring data feed any arbitrary collection of monitoring data (feed content), associated to an arbitrary keyword (feed name). A feed content can be any offline logged or real-time streaming information associated to a keyword, for instance the list of IP addresses (feed content) which are being querying a given DNS name (feed name), or a stream of events/alerts (feed content) associated to a cause (feed name). When unambiguous from the context, we will adopt the short notation feed to indicate either the feed content or the feed name.

We assume that the disclosure of which specific feed names are being tracked does not bring any privacy concerns (in other word, the employed feed names can be shared with no restriction), whereas we assume that the monitoring data associated to a feed, namely the feed content, is subject to strict confidentiality guarantees.

We do not pose any limit on the type and number of feeds being tracked. Actually, our goal is to develop a technique which may in principle scale up to hundred thousands or millions of very fine-grained feeds. One actual scenario motivating such goal is that of DNS-based Botnet detection, where, in the extreme case, one feed might be in principle associated to every individual DNS name being queried within the domain. Finally, we do not require feed names to be known beforehand, but any feed may start at any time (for instance, the first query to an unprecedented queried DNS name may start up such a new feed asynchronously).

\section{Data Sharing Model}

The data sharing model considered in this paper is graphically illustrated in figure 1 , for the special case of 4 domains and 7 feeds. We stress that while the number of domains is given, the number of feeds is neither assumed fixed nor known: for instance, in the case illustrated in the figure, any domain may asynchronously start a new feed at any time with no cross-domain coordination involved. 


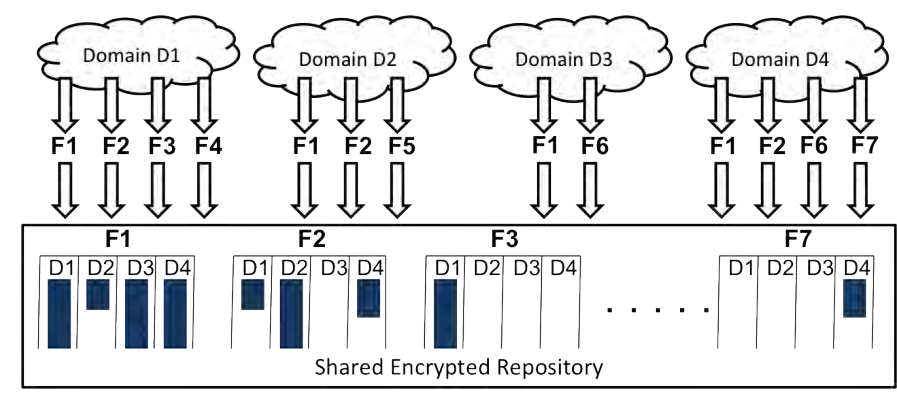

Fig. 1. Sharing Model

Each domain simply exports data organized into encrypted feeds, indexed by the plaintext feed name. Each feed is encrypted with a different key $K_{i, f}$, unique for the considered domain $i$ and the specific feed $f$. For scalability and performance reasons, we require very efficient and reliable encryption of the feed content. This can be accomplished through widely employed symmetric cipher suites, such as AES-CBC.

Once the data is exported, any collaborating domain may browse any data irrespective of whether it contributes or not to a given feed. However, it will be able to access the actual content only if it possesses the relevant encryption key. For simplicity of illustration, in figure 1 we depicted the case in which a shared physical repository collects all the data exported by all domains, but in practice this is only one among several alternatives. For instance, each domain may permit others to unrestrictedly access a local database accumulating the relevant per-domain feed material, or, alternatively, each domain may broadcast exported data to all remaining parties.

The content produced by different domains for a same feed name is further kept separate. Indeed, not only merging it would require a specific agreement on the content organization within every specific feed (agreement which is obviously crucial in practice, but which is not mandatorily assumed by our approach), but we also recall that content generated by different domains is encrypted with different keys.

\section{Design Requirements and Goals}

Our approach aims at satisfying three core requirements. First, a domain should be able to access all the data produced by all the domains for a given feed if an only if a threshold number of domains have granted permission to do so via a properly constructed cryptographic alert message. Our approach is constructed so as to enable all domains to access all data produced in a given feed, irrespective of whether a domain has actually contributed to provide contents to the feed, or whether it has actually raised an alert. The underlying rationale is that we aim at permitting sharing of data when global scale threats are detected, and for such types of threats we believe that access restrictions should not hold. Moreover, we believe that more elaborate schemes able to account for further restrictive conditions on the data access may be devised starting from the basic concepts and methods addressed in this paper.
Second, per-feed protection should be secure, in the meaning that a domain having given the ability to access a given subset of feeds should not have any advantage in getting access to the remaining feeds. Facing this requirement was the main design challenge at the start of this work. Indeed, with a small number of known feeds, the natural direction would have consisted in repeatedly applying standard threshold cryptography techniques [13], [14] for each deployed feed (i.e. sharing one dedicated and independent secret per feed). This of course is far from being viable in front of a potentially huge and unbounded number of originally unknown feeds, as it would require a coordination protocol on a per-feed basis and at every occurrence of a new feed. We succeed in overcoming this hurdle thanks to the intuition that a suitable combination of distributed threshold cryptosystems (in particular, Pedersen's Distributed Key Generation [15]) with Identity-Based Encryption (specifically Boneh-Franklin's pairing-based technique [16]) was an obvious (a posteriori!) way to address this requirement.

Finally, a third requirements is the full automatization of all the cryptographic key management framework (key generation and key reconstruction). Again, this is a mandatorily requirement in order to scale the system with the number of feeds. Our proposed solution leveraged ideas and primitives adapted to the network monitoring context in [11].

\section{TECHNICAL APPROACH}

\section{A. Intuition and Sketch}

Before entering into the detailed description of the procedures, we briefly and informally outline the basic steps and intuition behind our proposed construction.

Our approach is organized into two clearly distinguished phases: an offline phase, done once for all (irrespective of any feed specification), and an online phase composed of a sequence of steps applied, when needed, to every specific feed.

During the offline phase, the involved domains explicitly collaborate, via a suitably adapted Pedersen's Distributed Key Generation (DKG) scheme [15] to construct a unique shared master secret $s$. No single party gets to know such secret $s$. Rather, each party $i$ is provided with a share $x_{i}$ of the master secret $s$. Moreover, all parties get to know a public key $g^{s}$, derived from the master secret $s$. Such value is a point over a prime order elliptic curve group $G_{p}, g$ being the generator of such a group. The group $G_{p}$ is specifically chosen as the domain of a bilinear map $e: G_{p} \times G_{p} \rightarrow G_{T}$ suitable to be employed as pairing in the Boneh-Franklin's Identity Based Encryption (IBE) construction [16].

In essence, what just described is the set up of an infrastructureless $^{2}$. Identity Based Cryptosystem where, as usual, any party may asymmetrically encrypt a message for any "identity", by simply using just the public key $g^{s}$ and the

\footnotetext{
2 Indeed, no authority or player knows the master secret $s$, and thus, in contrast with traditional IBE frameworks, no centralized Private Key Generation (PKG) authority gets deployed. As such, it is worth to remark that our framework does not suffer of the deployment and security weaknesses frequently accounted to the need for a centralized PKG.
} 
identity's name, but where, initially, no party has the ability to decrypt anything!

The distinguishing feature of our approach is how we do exploit such a distributed identity-based cryptosystem. Our core idea consists in using feed names as "identities", i.e. public keys for IBE. Indeed, during the online phase, a domain $i$ exporting a feed $f_{I D}$ symmetrically encrypts it with a (pseudo-)random key $K_{i, f_{I D}}$. In addition, the domain exports $\mathcal{E}_{f_{I D}}\left(K_{i, f_{I D}}\right)$, i.e. the per-feed key $K_{i, f_{I D}}$ asymmetrically encrypted with IBE using as public key the same feed name.

No party may initially decrypt $\mathcal{E}_{f_{I D}}\left(K_{i, f_{I D}}\right)$ and hence access the feed, since the relevant private key $f_{I D}^{s}$ is not computable by any party (its computation requires knowledge of the master secret $s$, which we recall no single party has). However, every domain can trivially compute, at any time and for any feed name, a share $f_{I D}^{x_{i}}$ of such private key, using the feed name $f_{I D}$, and its private share $x_{i}$ of the master secret $s$ duly obtained during the offline phase. Therefore, reconstruction of the IBE private key, and in turns disclosure of all the set of keys $K_{i, f_{I D}}$ used by all the domains to encrypt the considered feed, is is trivially accomplished by combining a sufficient number of per-feed shares, $f_{I D}^{x_{i}}$, released by a sufficient number of domains $i$.

\section{B. Offline Setup}

We recall that, in a $(t, n)$ secret sharing scheme[13], a dealer splits a secret value into $n$ parts, called shares, and distributes such shares to the involved parties. Any combination of $t \leq n$ such shares permits to reconstruct the secret value.

The basic starting point in our proposed approach is the ability to share a secret across the cooperating domains, without relying on any central coordination authority or dealer. This problem has been first addressed in 1991 by Pedersen [15]. Pedersen's scheme further holds the advantage of being verifiable [17], i.e., it provides means to verify that the involved parties do not cheat the protocol rules. We do not explicitly include verifiability in the description of our proposed approach, as parties, being network operator domains, may be reasonably assumed honest-but-curious, but we recall that verifiability is trivially added to our construction by following [15].

The scheme used in our construction, and described in what follows, is based on the Pedersen's scheme duly adapted to our needs. Let $D_{i}, i \in(1, n)$, be the involved domains (parties). The parties preliminary agree on:

- two large primes $p$ and $q$ such that $q$ divides $p-1$;

- a threshold $t$, with $2 \leq t \leq n$, on the number of domains required to trigger feed decryption;

- a cyclic group $G_{p}$ of prime order $p$, and a generator $g \in$ $G_{p}$ for the group. Note that this group cannot be arbitrary but is (most likely) an elliptic curve, as it must be able to be used as domain of a bilinear map $e: G_{p} \times G_{p} \rightarrow G_{T}$ $\left(G_{T}\right.$ also being a cyclic group of order $p$ ) holding the properties described in the next section III-C.

Each party $D_{i}$ then performs the following steps:
1) chooses a random secret $\sigma_{i} \in Z_{q}$ over the ring of integers modulo prime $q$;

2) chooses a random polynomial of order $t-1$, $f_{i}(z)=\sigma_{i}+a_{1, i} z+\cdots+a_{t-1, i} z^{t-1} \in Z_{q}$, whose coefficients are elements of $Z_{q}$;

3) computes $s_{i, j}=f_{i}(j) \bmod q$ for $j \in(1, n)$, and sends to every party $D_{j}$ the value $s_{i, j}$ via a secure unicast communication;

4) computes $g^{\sigma_{i}} \in G_{p}$, and broadcasts it to all the remaining parties.

Let us now define as master secret the quantity $s=\sum_{i=1}^{n} \sigma_{i}$. At the end of these steps, no domain has knowledge of the shared master secret $s$, but each domain is able to compute two crucial quantities:

- the global public key $g^{s}=\prod_{i=1}^{n} g^{\sigma_{i}} \in G_{p}$;

- the party $i$ local share $x_{i}=\sum_{j=1}^{n} s_{i, j} \bmod q$.

The system is secure as long as the master secret $s$ remains unknown to all the involved parties and to attackers; as well known, this holds if and only if no more than $t-1$ parties collude. Against honest-but-curious adversaries, the scheme has the further additional property that the master secret is uniformly distributed in $Z_{q}$, and no information can be learned by an adversary ${ }^{3}$, except for what is implied by the knowledge of the public value $g^{s}$.

\section{Online feed management primitives}

Our proposed construction relies on properties which are found in Identity Based Cryptosystem developed by Boneh and Franklin [16], actually the first fully functional IBE proposed. This scheme is based on bilinear maps $e: G_{p} \times G_{p} \rightarrow G_{T}$ between two cyclic groups of same prime order $p$. An applicable map must satisfy the following properties:

- bilinear: for all $u, v \in G_{p}$ and all $a, b \in \mathcal{Z}$, $e\left(u^{a}, v^{b}\right)=e(u, v)^{a b}$

- non degenerate: the map should not send all pairs to the identity in $G_{T}$; rather, being $g$ a generator of $G_{p}$, then $e(g, g)$ must generate $G_{T}$.

- computable: there is an efficient algorithm to compute any pairing.

The Weil (used in [16]) or Tate pairing [19] on elliptic curve groups are well known and exploited examples of admissible maps. Given such a pairing primitive, a (basic) BonehFranklin's IBE is constructed as follow. Let $H:\{0,1\}^{*} \rightarrow G_{p}^{*}$ be a cryptographic hash function which maps any arbitrary string over a non unitary point in $G_{p}$, and $H_{2}: G_{T} \rightarrow\{0,1\}^{h}$ another hash function which maps a point in $G_{T}$ over a string of some length $h$. Let $g^{s} \in G_{p}$ be the overall public key, and $s$ be the relevant master secret (both discussed in the previous section).

\footnotetext{
${ }^{3}$ We remark that this does not hold any more against malicious adversaries: an active attacker controlling some parties may succeed in biasing the generated secret away from the expected uniform distribution [18]. It does not appear trivial to integrate the more secure approach [18] in our proposed construction and, besides, this weakness emerges only in the presence of malicious parties, and hence does not does not appear to be critical in our network operators' scenario.
} 
Encryption $\mathcal{E}_{I D}(M)$ - To encrypt a message $M$ using as public key a string $I D$, choose a random value $r \in Z_{q}^{*}$; the ciphertext is the pair of values

$$
\mathcal{E}_{I D}(M)=\left\langle g^{r}, M \oplus H_{2}\left(e\left(H(I D)^{r}, g^{s}\right)\right)\right\rangle
$$

where we note that, owing to the bilinear property of the pairing, $e\left(H(I D)^{r}, g^{s}\right)=e(H(I D), g)^{r s}$.

Decryption - Decryption requires knowledge of a private key $H(I D)^{s} \in G_{p}$ associated to the string $I D$. While this is typically provided by a trusted party called Private-Key Generator (PKG) who knows the master secret $s$, in our approach construction of such private key is jointly done by the involved parties as discussed later. Once such private key is known, decryption simply consists in computing:

$$
M=\mathcal{E}_{I D}(M) \oplus H_{2}\left(e\left(H(I D)^{s}, g^{r}\right)\right)
$$

Indeed, $e\left(H(I D)^{s}, g^{r}\right)=e(H(I D), g)^{r s}$, hence the same point in $G_{T}$ used to construct the ciphertext is reobtained.

Building on these bases, our proposed online handling of feeds can be summarized into two classes of tasks: those related to feed encryption, and those related to per-feed key reconstruction.

\section{Feed encryption}

Since feed content may be bulky, obvious performance reasons recommend its encryption via off-the-shelf efficient symmetric ciphersuites (e.g., AES). To automatically manage differentiated per-feed encryption and cope with the lack of a-priori knowledge about possible feeds, we suggest to derive keys by applying (keyed) pseudo-random functions (PRFs)[20] to the feed name. Indeed, PRF-based key derivation is customary in network security protocols such as IPsec or TLS. Following [11], we specifically derive the symmetric key which domain $D_{i}$ shall apply to a feed whose name is $f_{I D}$ as

$$
K_{i, f_{I D}}=\operatorname{PRF}\left(S_{i}, f_{I D}\right)
$$

where $S_{i}$ is a domain-specific local random secret. Note that the so-generated per-feed keys do not require to be stored (i.e. they are stateless); indeed, (3) may be recomputed every time new feed content is to be exported (we assume that the feed name can be obtained from the monitoring data to be exported - for instance this is the case when the feed name is a DNS name, a flow tuple, a network prefix, etc.).

Moreover, to permit feed decryption (as described below), whenever a new feed is started (or found), an asymmetrically IBE-encrypted version of the per-feed key, $\mathcal{E}_{f_{I D}}\left(K_{i, f_{I D}}\right)$ must be broadcast to the remaining domains (e.g. stored in the shared repository of figure 1). Note that this operation, computationally costly, at least in principle may be done only once per every feed; in practice periodic refreshes of such encrypted key transmission may improve reliability.

\section{Per-feed key reconstruction}

Goal of our approach is to permit all domains to access all the contents associated to a specific feed, when a threshold number of domains signal such feed as anomalous. To this purpose, each domain is in charge of locally monitoring feeds, and responsible of deciding under which specific conditions a feed should be signalled as anomalous; such decision is specific to each feed semantics, and as such it is out of the scope of this paper.

Assume that a domain $D_{i}$ takes such decision on a given feed having name $f_{I D}$. Then, the domain broadcasts the value $H\left(f_{I D}\right)^{x_{i}} \in G_{p}$. This value is a share of the IBE private key associated to the feed name $f_{I D}$, but does not disclose any information on the remaining feeds, as the domain's share $x_{i}$ of the master secret $s$ remains private.

Whenever $t$ or more shares $H\left(f_{I D}\right)^{x_{i}}$ are made public, irrespective of whether it has contributed with a share, any of the $n$ domains may restore the IBE private key $H\left(f_{I D}\right)^{s}$ for the feed $f_{I D}$, via (exponent) Lagrange interpolation:

$$
H\left(f_{I D}\right)^{s}=\prod_{k \in \mathcal{Q}}\left(H\left(f_{I D}\right)^{x_{k}}\right)^{\Lambda_{k}}=H\left(f_{I D}\right)^{\sum_{k \in \mathcal{Q}} x_{k} \Lambda_{k}}
$$

where $\mathcal{Q}$ is the set of indices of the $t$ domains releasing their share, and $\Lambda_{k}$ is the Lagrange coefficient

$$
\Lambda_{k}=\prod_{j \in \mathcal{Q} \backslash\{k\}} \frac{j}{j-k}
$$

The knowledge of feed $f_{I D}$ 's private key $H\left(f_{I D}\right)^{s}$ permits to decrypt all the values $\mathcal{E}_{f_{I D}}\left(K_{i, f_{I D}}\right)$ made available by the domains contributing to the considered feed, hence access all the relevant feed' symmetric encryption keys and decrypt all past and future exported feed content.

Finally, concerning security, although a formal analysis is out of the scopes of this preliminary work, we at least remark that our construction inherits the security properties of [16]; specifically an attacker who has been revealed the private keys for an arbitrary number of feed names is not able to mount an attack devised to obtain the private key for a new feed.

\section{Preliminary Implementation Remarks}

In order to verify the correct functional operation of the proposed approach, we developed the relevant cryptographic functions in software and assessed their performance.

For what concerns symmetric encryption and pseudorandom function, we used the implementation available in the widely employed OpenSSL library of the Advanced Encryption Standard cipher suite AES-128, with 128 bits key, and of the HMAC SHA-256 PRF, respectively.

Concerning pairing, elliptic curve generation, elliptic curve arithmetic, and hash functions required by IBE, we leveraged the primitives made available by the public domain Pairing Based Cryptography (PBC) library [21], which in turns uses the GMP library [22]. We specifically used the type-A pairing provided in PBC, which uses the elliptic curve $y^{2}=x^{3}+x$ over the field $Z_{p}$ for some prime $p$.

The offline setup phase, based on the suitably adapted Pedersen's DKG scheme, was written from scratch and was partly based on the PBC library as well, since, in our specific construction, the public terms used to derive the public key are elliptic curve points. 
TABLE I

EXPERIMENTAL PERFORMANCE MEASUREMENTS - PER FUNCTION BREAKDOWN

\begin{tabular}{|l|c|}
\hline Function & Performance(ms) \\
\hline IBE encryption, eq. 1 & 13.49 \\
IBE decryption, eq. 2 & 13.08 \\
Symmetric key derivation, eq.3 & 0.009 \\
Computation of IBE Private Key share & 5.46 \\
Lagrange interpolation, eq.4, 3 Parties & 16.78 \\
Lagrange interpolation, eq.4, 5 Parties & 29.45 \\
Lagrange interpolation, eq.4, 10 Parties & 55.92 \\
AES-128 packet encryption & 0.015 \\
\hline
\end{tabular}

Our preliminary tests focused on the quantification of the computational demand required by the online operations which characterize our scheme. Results are produced by processing IP packets of size 1500 bytes, using as feed name the pair (IP source, IP destination), and using as feed content the whole packet. Performance, summarized in table I, and obtained on a PC with Intel CPU at $2.40 \mathrm{GHz}$ frequency, quantify the time needed to perform each function, averaged over 3000 different packets (i.e., each new packet is assumed to instantiate a new feed). As well expected, such results show that the most expensive operation are the Lagrange interpolation and the IBE-related computations, especially those involving pairings.

A thorough understanding of the relevant implications on scalability would require to assess our approach over a realistic setting, specifying how monitoring data feeds are defined and under which conditions feed contents should be disclosed, an activity planned for future work. But a few preliminary considerations can be nevertheless drawn.

We first remark that per-feed content encryption and symmetric key derivation are the only bulky tasks, being persistently applied to any exported feed content. However, they can be relatively easily offloaded via hardware implementation [12] and scaled to gbps speed. Concerning IBE tasks, note that IBE encryption of the symmetric key is applied only once per feed, so its impact depends on the frequency of generation of new feeds. Results presented in table I suggest that support of a few hundred thousand new monitoring data feeds per hours may be well at reach. Finally, all the remaining IBE-related operations are even more rare, as they are only performed in conjunction with the emergence of anomalies.

\section{CONCLUSION}

This work has presented a cryptographic scheme devised to support a cooperative cross-domain monitoring functionality referred to as conditional data sharing, which consists in permitting sharing of fine-grained organized data only when a threshold number of parties are ready to reveal their data. The proposed approach does not require any explicit cooperation besides an once-for-all initial setup phase, and does not pose any in principle restriction on how data are organized into socalled feeds, and on how many (and when) feeds are deployed.

The current work is preliminary and limited to the presentation of the cryptographic framework. Ongoing work is focusing on the validation of our framework on realistic crossdomain network monitoring scenarios, among which a first candidate use case is the sharing of bot identities associated to a same set of fast-fluxing DNS feed names.

Moreover, planned future work include the extension of the system to accommodate more flexible sharing policies that go beyond the basic threshold approach currently supported.

\section{ACKNOWLEDGEMENT}

This work was partially supported by DEMONS, a research project supported by the European Commission under its 7 th Framework Program (contract no. 257315).

\section{REFERENCES}

[1] P. Porras and V. Shmatikov, "Large-scale collection and sanitization of network security data: risks and challenges," in Proc. 2006 workshop on New security paradigms. NY, USA: ACM, 2007, pp. 57-64.

[2] F. Huici and M. Handley, "An edge-to-edge filtering architecture against dos," SIGCOMM Comp. Commun. Rev., vol. 37, pp. 39-50, March 2007.

[3] V. Gowadia, E. Scalavino, E. C. Lupu, D. Starostin, and A. Orlov, "Secure cross-domain data sharing architecture for crisis management," in Proc. 10-th ACM workshop on Digital rights management (DRM '10), Chicago, IL, USA, 2010, pp. 43-46.

[4] ENISA - European Network and Information Security Agency, "Botnets: Detection, measurement, disinfection and defence," 2011.

[5] F. Gaudino and G. V. Lioudakis, "Interjurisdictional privacy compliance," FP7 DEMONS project, Deliverable D2.2, 2011.

[6] G. V. Lioudakis, F. Gaudino, E. Boschi, G. Bianchi, D. I. Kaklamani, and I. S. Venieris, "Legislation-aware privacy protection in passive network monitoring," in Information Communication Technology Law, Protection and Access Rights: Global Approaches and Issues, I. M. Portela and M. M. Cruz-Cunha, Eds. IGI Global, 2010, ch. 22, pp. 363-383.

[7] R. Pang, M. Allman, V. Paxson, and J. Lee, "The devil and packet trace anonymization," SIGCOMM Comp. Commun. Rev., pp. 29-38, 2006.

[8] M. Foukarakis, D. Antoniades, S. Antonatos, and E. P. Markatos, "On the anonymization and deanonymization of netflow traffic," in Proc. of FloCon 2008, Savannah, USA, Jan. 2008.

[9] M. Burkhart, D. Schatzmann, B. Trammell, E. Boschi, and B. Plattner, "The role of network trace anonymization under attack," SIGCOMM Comp. Commun. Rev., vol. 40, no. 1, pp. 5-11, jan 2010.

[10] P. Ohm, "Broken promises of privacy: Responding to the surprising failure of anonymization," UCLA Law Review, vol. 57, 2009.

[11] G. Bianchi, S. Teofili, and M. Pomposini, "New directions in privacypreserving anomaly detection for network traffic," in Proc. 1st ACM workshop on Network data anonymization (NDA '08), 2008, pp. 11-18.

[12] G. Bianchi, J. Wolkersdorfer, C. Greco, I. Gojmerac, and S. Teofili, "Feasibility of wire-speed hardware-based conditional per-flow encryption for on-the-fly protection of monitored traffic," in Future Networks \& Mobile Summit 2010, June 2010.

[13] A. Shamir, "How to share a secret," Commun. ACM, vol. 22, pp. 612613, Nov 1979.

[14] G. R. Blakley, "Safeguarding cryptographic keys," Managing Requirements Knowledge, International Workshop on, p. 313, 1979.

[15] T. P. Pedersen, "A threshold cryptosystem without a trusted party," in Proc. 10th int. conf. on Theory and application of cryptographic techniques, ser. EUROCRYPT'91, 1991, pp. 522-526.

[16] D. Boneh and M. K. Franklin, "Identity-based encryption from the weil pairing," in Advances in Cryptology - CRYPTO'01, pp. 213-229, 2001.

[17] B. Chor, S. Goldwasser, S. Micali, and B. Awerbuch, "Verifiable secret sharing and achieving simultaneity in the presence of faults," in Proc. 26th IEEE Symp. on Foundations of Computer Science, 1985, pp. 383 395.

[18] R. Gennaro, S. Jarecki, H. Krawczyk, and T. Rabin, "Secure distributed key generation for discrete-log based cryptosystems," Journal of Cryptology, Springer, vol. 20, pp. 51-83, 2007.

[19] A. Joux, "The weil and tate pairings as building blocks for public key cryptosystems," Proc. 5th Int. Symp. on Algorithmic Number Theory, Springer-Verlag, vol. 2369, pp. 11-18, 2002.

[20] N. Williams, "A pseudo-random function api extension for the generic security service application program interface," RFC 4401, 2006.

[21] B. Lynn, "Pairing-based cryptography," http://crypto.stanford.edu/pbc/.

[22] T. Granlund, "The gnu multiple precision arithmetic library," http://gmplib.org/. 